\title{
Perfil Epidemiológico Quirúrgico del Hospital Universitario San José de Popayán
}

\section{Epidemiologic Surgical Profile of the Hospital Universitario San José, Popayan}

\author{
Valentina Agredo-Delgado', Jesús David Álvarez-Morales', Ginna Belalcazar-Hernández', \\ Alexis Berrio-Chavarria', Johanna Betancourt-Cañas', Daniel Bolaños-Ñañez', \\ Juan Pablo Caicedo², José Andrés Calvache*
}

\begin{abstract}
Resumen
Introducción: La cirugía es parte integral de la atención en salud y es un procedimiento diagnóstico y terapéutico al que un gran porcentaje de la población se ha sometido en algún momento de su vida. Anualmente se hacen a nivel mundial 234 millones de procedimientos quirúrgicos. El objetivo de este estudio fue describir y analizar la distribución epidemiológica de todas las cirugías realizadas en el año 2014 en el Hospital Universitario San José de Popayán, Colombia (HUSJ). Metodología: Estudio observacional de corte transversal. Los datos fueron tomados de forma directa de la base de datos de dirección de quirófanos y área de sistemas del HUSJ durante el año 2014 previa aprobación del Comité de Ética Institucional. Se construyeron categorías para las variables edad y especialidad quirúrgica. Se analizó mediante estadística descriptiva y gráfica. Resultados: 11.090 pacientes se sometieron a cirugía en el periodo en estudio. El $50,5 \%$ de los pacientes fueron de género femenino. La especialidad con mayor frecuencia quirúrgica fue cirugía adultos con 3044 procedimientos $(27 \%)$; seguida de cirugía plástica, ginecobstetricia y ortopedia y traumatología cada una con aproximadamente 1400 procedimientos (12\%). Los procedimientos hospitalarios representaron el 34\%, cirugías de urgencias (33\%) y cirugía ambulatoria $32 \%$. El promedio global de tiempo quirúrgico en el total de procedimientos fue de 58 minutos (1DE=41) con una mediana de 47 minutos RIQ [30-70]. Discusión: La distribución quirúrgica del HUSJ en el año 2014 tiene concordancia con reportes de otras latitudes para niveles de complejidad similar. Diferencias regionales representan oportunidades de mejoramiento y priorización en los procesos relacionados.
\end{abstract}

Palabras Clave: Perfil de salud, prestación de asistencia sanitaria, cirugía, especialidad (Fuente: DeCS).

\begin{abstract}
Introduction: Surgery is an integral part of health care and it is a diagnostic and therapeutic procedure that a large proportion of the population has undergone at some point in their life. They are made annually worldwide 234 million surgical procedures. Objective: The objective of this study was to describe and analyze the epidemiological profile and distribution of all surgeries performed in 2014 at the Hospital Universitario San José, Popayán, Colombia (HUSJ). Methodology: Cross-sectional study. The data were taken directly from the hospital database and system data area during 2014 after approval by the institutional ethics committee. There were constructed categories for age and surgical specialty. Descriptive statistics and graphics were done. Results: 11.090 patients underwent surgery in the period under study.
\end{abstract}

1 Universidad del Cauca, Facultad Ciencias de la Salud, Estudiantes programa de Medicina.

2 Universidad del Cauca, Facultad Ciencias de la Salud, Profesores Departamento de Anestesiología.Erasmus University Medical Centre, Department of Anesthesiology, Rotterdam, The Netherlands.

* Correspondencia: MD. José Andrés Calvache. Correo electrónico: jacalvache@unicauca.edu.co

Recibido: 28/01/2016 - Revisado: 03/03/2016 - Aceptado: 08/05/2016 
$50,5 \%$ of patients were female. Surgical specialty more often was adult surgery with 3044 procedures $(27 \%)$; followed by plastic surgery, gynecology and orthopedics and traumatology each with approximately 1400 procedures $(12 \%)$. Hospital procedures were $34 \%$, emergency surgeries $(33 \%$ ) and outpatient surgery $32 \%$. The overall average operating time was 58 minutes $(I S D=41)$ with a median of 47 minutes IQR [30-70]. Discussion: Surgical distribution at HUSJ in 2014 is consistent with reports from other regions to levels of similar complexity. Regional differences represent opportunities for improvement and prioritization in related processes.

Keywords: Health profile, Delivery of Health Care, Surgery, Specialization (Source: DeCS).

\section{Introducción}

La cirugía es parte integral de la atención en salud y es un procedimiento diagnóstico y terapéutico al que un gran porcentaje de la población se ha sometido en algún momento de su vida. Anualmente se hacen a nivel mundial 234 millones de procedimientos quirúrgicos. Según el Banco Mundial, el 11\% de la carga de enfermedad es tratable con cirugía, lo que equivale a 164 millones de años de vida por discapacidad. Además, ningún grupo poblacional está exento de la necesidad de este tipo de procedimiento ${ }^{(1)}$.

Diversos países como India, Chile, Estados Unidos, entre otros, han determinado claramente el perfil epidemiológico quirúrgico de sus poblaciones ${ }^{(1,2)}$. En Colombia, se han realizado estudios en subespecialidades quirúrgicas ${ }^{(3)}$, pero en nuestro conocimiento, este es el primer estudio que se concentra en el volumen total quirúrgico de un hospital universitario.

Ante la gran y creciente demanda de esta intervención en salud, surge la necesidad de conocer esos datos y sus distribuciones en nuestro medio. El objetivo del presente estudio fue describir y analizar la distribución epidemiológica de todas las cirugías realizadas en el año 2014 en el Hospital Universitario San José de Popayán, Cauca.

\section{Metodología}

Estudio observacional de corte transversal. Los datos fueron recolectados de forma directa a partir de la base de datos quirúrgica del año 2014 existente en la Dirección de Quirófanos y Área de Sistemas del HUSJ previa aprobación del protocolo de investigación por parte la coordinación de esta unidad administrativa y del Comité de Ética Hospitalario.

Se consideraron a todos pacientes intervenidos quirúrgicamente en el Hospital Universitario San José durante el año 2014 (1 de enero a 31 de diciembre de 2014) para lograr potencial inclusión. Sin embargo el único criterio de inclusión adicional utilizado fue que los datos epidemiológicos y clínicos estuviesen completos en la base de datos para cada unidad de análisis (paciente individual). Para los pacientes que presentaban múltiples cirugías en una única hospitalización se consideró únicamente el primer procedimiento para el análisis.

La estructura de la base de datos inicial presentaba múltiples unidades de análisis por paciente, puesto que generaba una fila para cada procedimiento codificado de acuerdo al manual de procedimientos quirúrgicos del HUSJ. Para su recodificación y compilación de la unidad de análisis se consideró el procedimiento principal descrito como el primero del listado (en caso de múltiples procedimientos). Este procedimiento se realizó en el paquete estadístico $\mathrm{R}^{(4)}$.

La base de datos se analizó mediante el paquete estadístico R. Se construyeron categorías para las variables edad y especialidad quirúrgica. Los análisis se hicieron en su mayoría mediante estadística descriptiva y gráficas. Se utilizaron medidas de resumen numérico como frecuencias y proporciones y medidas de centralización y dispersión de acuerdo a la distribución de las variables.

\section{Resultados}

Pacientes: En el HUSJ 11.090 pacientes se sometieron a cirugía durante el año 2014. El 49,5\% (5.487) pacientes fueron de género masculino y $5.603(50,5 \%)$ de género femenino. El grupo operado con mayor frecuencia fueron los "adultos" correspondiente a los pacientes comprendidos entre los 18 y los 64 años, y su frecuencia fue del $63.9 \%$. Respecto al régimen de afiliación a salud, los pacientes más frecuentes fueron los afiliados al régimen subsidiado con el 51,7\% de los casos, seguido del contributivo con el $33,4 \%$ de los casos (Tabla 1).

Procedimientos quirúrgicos: En el Hospital Universitario San José durante el año 2014 se practicaron 678 cesáreas correspondientes al $6.1 \%$, constituyéndose como el procedimiento más frecuentemente. La Tabla 2 presenta la distribución de frecuencias de los procedimientos.

El tipo de herida más frecuente fue de tipo "limpia" en $57,4 \%$. Seguida de "limpia contaminada" en $33,2 \%$ de los casos. La frecuencia acumulada entre los dos tipos de herida más frecuentes fue $90,6 \%$. La herida menos frecuente fue la de tipo "sucia" con el 3,7\% de los casos.

Del total de procedimientos, la mayoría de los pacientes fueron cirugías de tipo hospitalario (34\%), seguido de cirugías de urgencias (33\%). Los pacientes ambulatorios constituyeron un $(32 \%)$.

Diagnósticos y especialidades: Los cinco principales diagnósticos preoperatorios en su orden fueron: hiperplasia prostática (226 pacientes), pterigion (195 pacientes), apendicitis aguda no especificada (182 pacientes), heridas de otras partes de la cabeza (177 pacientes) y dolor crónico intratable (175 pacientes). 
Tabla 1. Características generales y demográficas de los pacientes sometidos a cirugía en el HUSJ en el año 2014 $(n=11.090)$

\begin{tabular}{|c|c|c|}
\hline \multirow{2}{*}{ Variable en estudio } & \multicolumn{2}{|c|}{ Media \pm 1 de frecuencia $(\%)$} \\
\hline & \multicolumn{2}{|c|}{ Media $42 \pm 23$} \\
\hline $\begin{array}{l}\text { Adulto mayor (mayor a } 65 \text { años) } \\
\text { Adulto (entre } 18 \text { y } 64 \text { años) } \\
\text { Adolescente (entre } 12 \text { y } 17 \text { años) } \\
\text { Escolar (entre } 6 \text { y } 11 \text { años) } \\
\text { Preescolar (entre } 2 \text { y } 5 \text { años) } \\
\text { Lactante (menor de } 2 \text { años) }\end{array}$ & $\begin{array}{c}2.395(21,6) \\
7.086(63,9) \\
532(4,8) \\
454(4,1) \\
399(3,6) \\
224(2,1)\end{array}$ & $\begin{array}{l}(21,6) \\
(63,9) \\
(4,8) \\
(4,1) \\
(3,6) \\
(2,1)\end{array}$ \\
\hline \multicolumn{3}{|c|}{ Régimen de afiliación } \\
\hline $\begin{array}{c}\text { Subsidiado } \\
\text { Contributivo } \\
\text { Régimen Especial* } \\
\text { SOAT } \\
\text { Vinculado } \\
\text { Particular }\end{array}$ & $\begin{array}{l}5735 \\
3707 \\
623 \\
567 \\
286 \\
172\end{array}$ & $\begin{array}{l}(51,7) \\
(33,4) \\
(5,6) \\
(5,1) \\
(2,6) \\
(1,6)\end{array}$ \\
\hline
\end{tabular}

* Fuerzas Armadas, Magisterio de profesores.

Tabla 2. Diez procedimientos quirúrgicos más realizados en el HUSJ en el 2014 ( $n=11.090)$

\begin{tabular}{lll}
\hline Codificación SOAT & Frecuencia (\%) \\
\hline Cesárea & 678 & $(6,1)$ \\
Desbridamiento de tejidos blandos $<5 \%$ profundo & 358 & $(3,2)$ \\
Instalación bomba de infusión para dolor (Catéter epidural) & 332 & $(3)$ \\
Colecistectomía por laparoscopia & 297 & $(2,7)$ \\
Colgajo muscular miocutáneo y fasciocutáneo & 293 \\
Instalación de catéter subclavio, femoral, yugular o peritoneal por punción & 280 \\
Bloqueo simpático por regiones & 264 & $(2,4)$ \\
Cistoscopia y calibración uretral & 235 & $(2,1)$ \\
Sección y ligadura de Trompa de Falopio & 219 & $(2)$ \\
Venodisección y catéter subclavio & 199 & $(1,8)$ \\
\hline
\end{tabular}




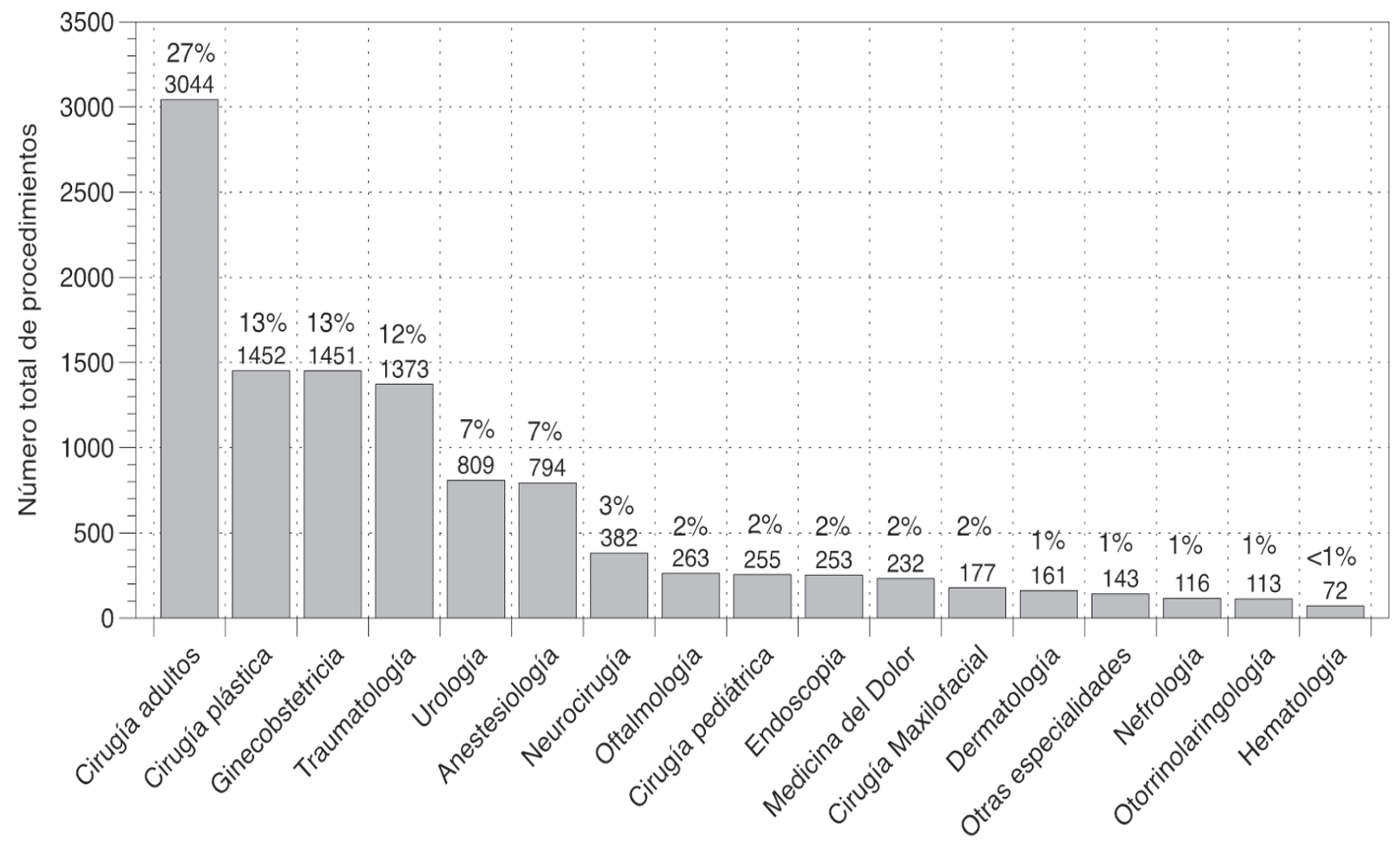

Figura 1. Distribución quirúrgica del número de procedimiento realizados en el año 2014 en el HUSJ por especialidad tratante $(\mathrm{n}=11.090)^{*}$

* Cirugía adultos: Categoría consolidada por Cirugía abdominal, laparoscópica, torácica y cardiovascular (de acuerdo a la estructura de datos de HUSJ).

La distribución quirúrgica del año 2014 por especialidad tratante se presenta en la Figura 1. La especialidad quirúrgica que presenta una mayor frecuencia es cirugía adultos con 3044 procedimientos $(27 \%)$, seguida de cirugía plástica, ginecobstetricia y ortopedia y traumatología cada una con aproximadamente 1400 procedimientos (12\%) cada una. El equipo "intensificador de imagen" se utilizó en un $10 \%$ del total de procedimientos.

Procedimientos anestésicos: El tipo de anestesia más utilizado fue anestesia general en 5.253 pacientes $(47,4 \%)$, seguida en frecuencia de anestesia neuroaxial raquídea 2.547 pacientes (23\%) y anestesia local en $2114(19 \%)$. En menores proporciones se usó bloqueos regionales y sedación.

Tiempo de duración procedimientos quirúrgicos: Para realizar análisis se seleccionaron los datos comprendidos entre el percentil 3 (10 minutos) y 97 (360 minutos) que correspondieron a 10.505 casos. El promedio global de tiempo quirúrgico en el total de procedimientos fue de 58 minutos ( $1 \mathrm{DE}=41$ ), con una mediana de 47 minutos RIQ [30-70]. La Figura 2 presenta la distribución del tiempo quirúrgico para las ocho especialidades con mayor frecuencia de procedimientos $(\mathrm{n}=9.066)$.
Tendencias temporales: En el HUSJ, durante el mes de julio del año 2014 se presentó la mayor frecuencia de procedimientos quirúrgicos con 1.134 cirugías equivalentes al 10.2\% de las cirugías totales; seguido por el mes de agosto con 993 $(9.0 \%)$. La Figura 3 presenta la frecuencia diurna y nocturna de realización de procedimientos.

Del total de procedimientos, 9.187 cirugías fueron en horario diurno (7am-7pm) (83\%) y 1.903 en horario nocturno $(17,2 \%)$. La figura 4 presenta la frecuencia de cirugías realizadas de acuerdo al día de la semana. Del total de cirugías, $84,5 \%$ (9.367 casos), 1.723 (15,5\%) se realizaron en fin de semana (sábado o domingo). La Figura 5 muestra el número total de procedimientos realizados por días de la semana en el HUSJ en el año 2014.

\section{Discusión}

Los principales hallazgos de estudio son: i) la especialidad de cirugía general (y sus subespecialidades) es la que más frecuentemente realiza procedimientos quirúrgicos en el HUSJ, seguida por cirugía plástica, traumatología y ginecobstetricia. ii) la atención quirúrgica del HUSJ se distribuye de forma 


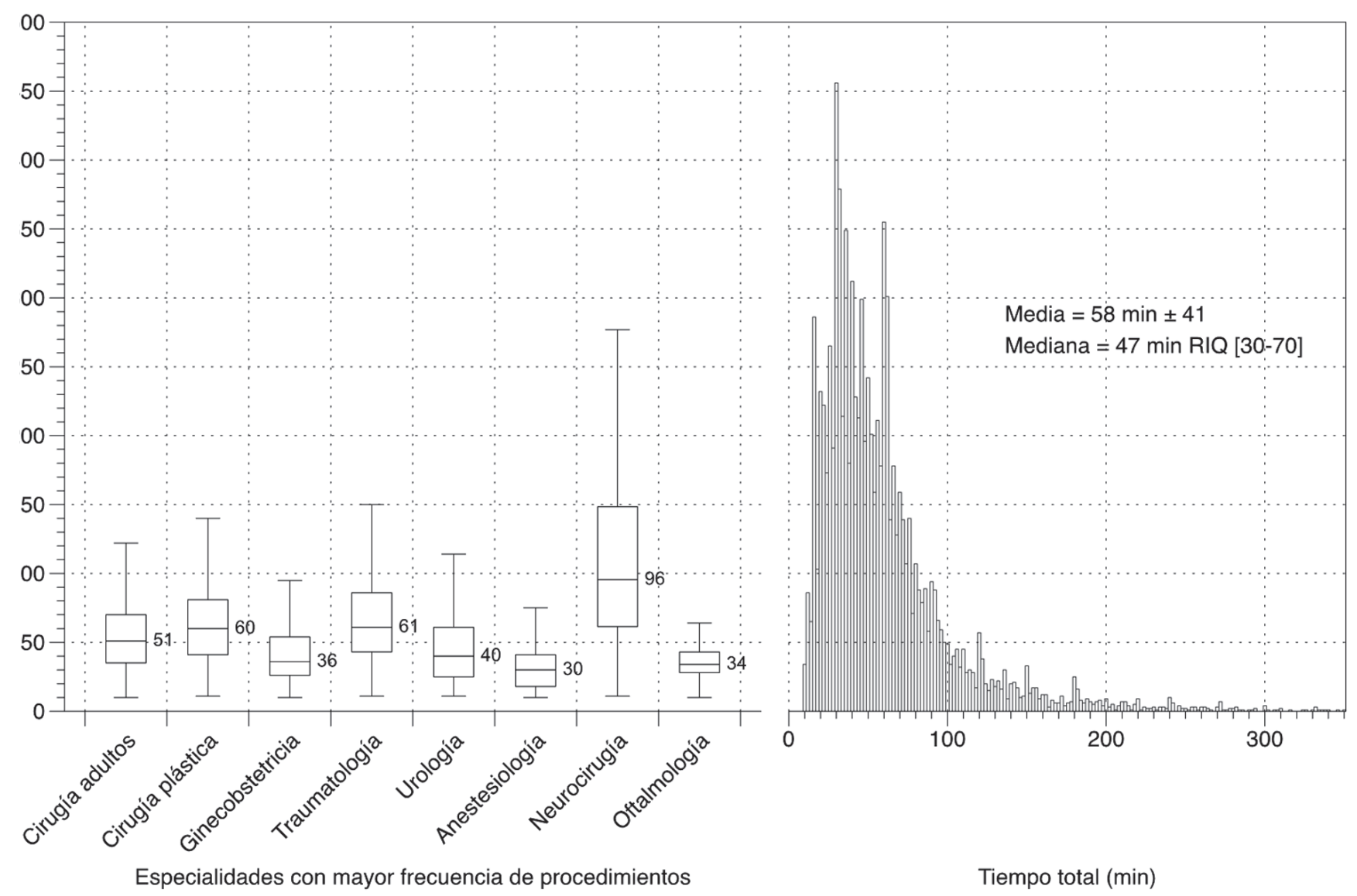

Figura 2. Tiempo quirúrgico de procedimientos realizados en el año 2014 en el HUSJ por ocho especialidades de mayor número de cirugías $(\mathrm{n}=9.066)$

* El valor al lado de los diagramas de caja representa la mediana de duración de cada especialidad.

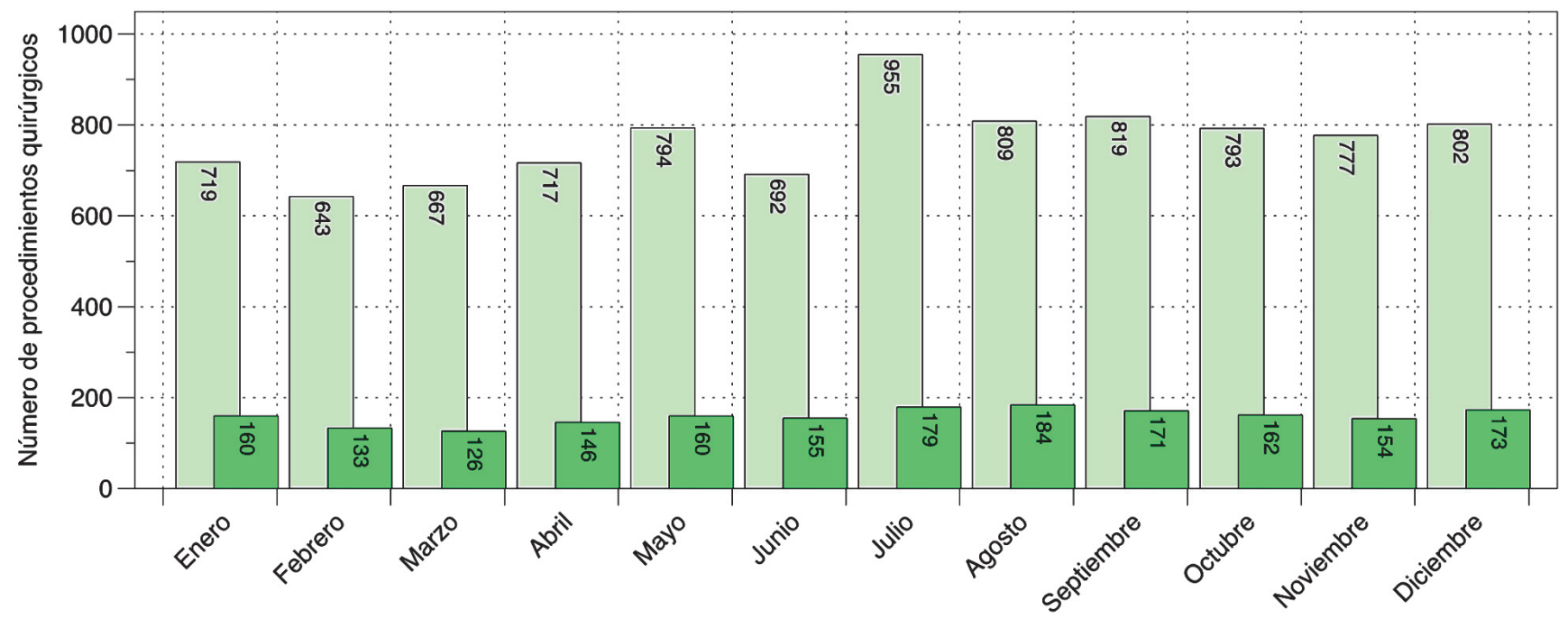

Figura 3. Frecuencia diurna y nocturna por mes de realización de procedimientos quirúrgicos en el año 2014 en el HUSJ $(n=11.090)$. 


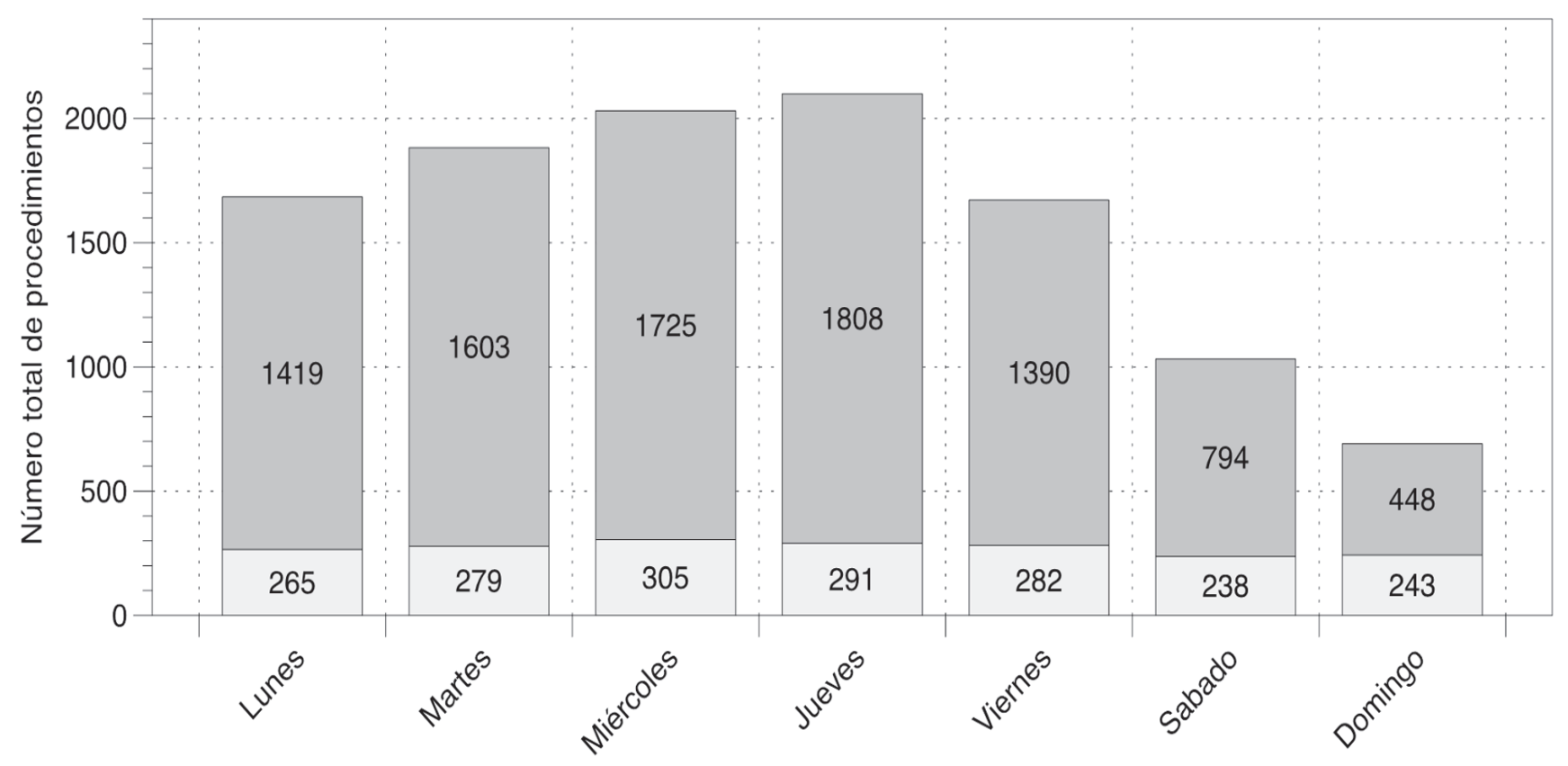

Figura 4. Frecuencia diurna, nocturna y por día de la semana de realización de procedimientos quirúrgicos en el año 2014 en el HUSJ $(n=11.090)$

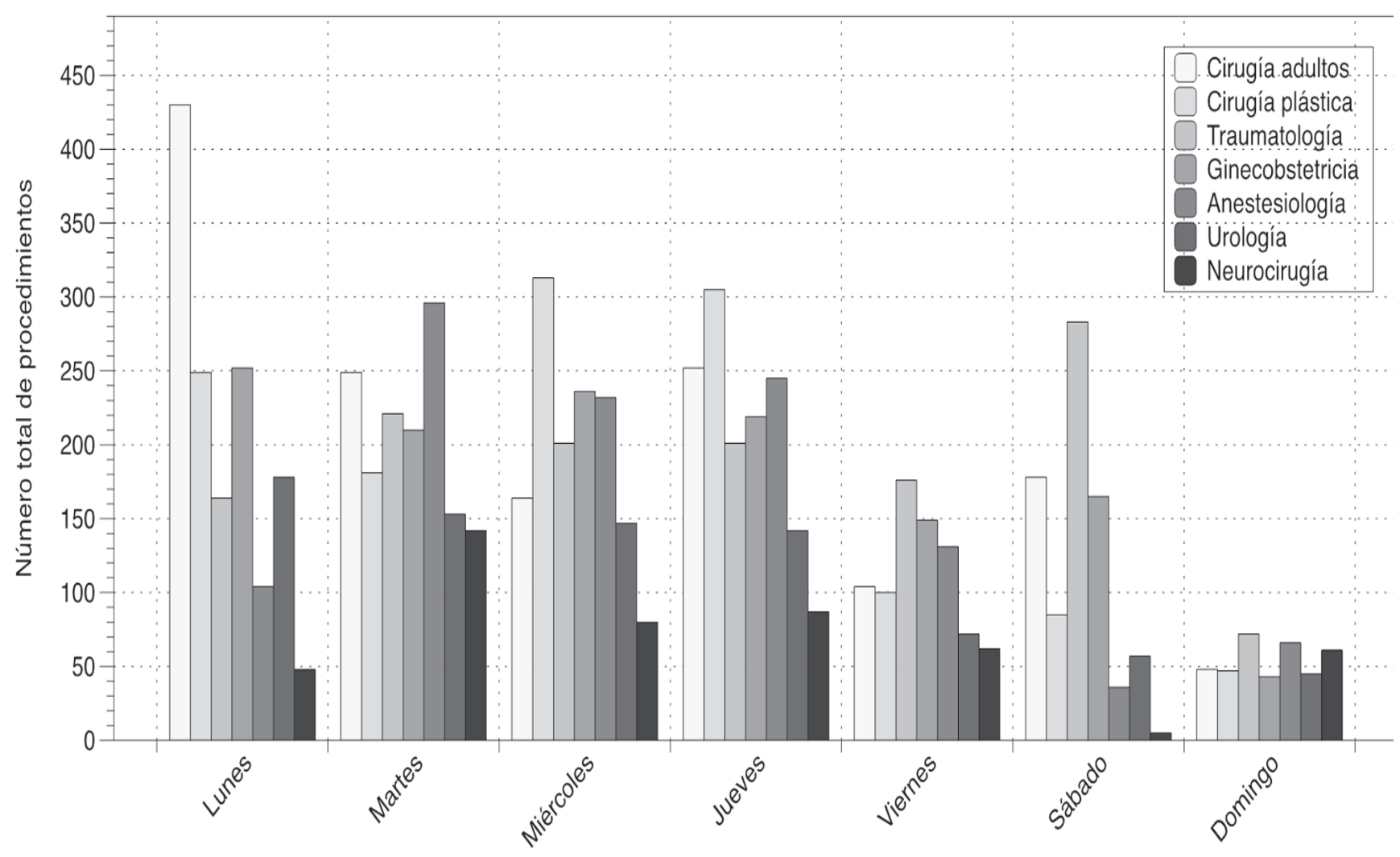

Figura 5. Número total de procedimientos realizados por días de la semana por siete especialidades con mayor utilización de quirófano en el año 2014 en el HUSJ $(n=11.090)$. 
equitativa entre las categorías de cirugía de paciente hospitalizado, urgencias y ambulatoria. iii) la mayor cantidad de pacientes operados en HUSJ son adultos pertenecientes al régimen subsidiado y iv) la mediana consolidada de duración quirúrgica es de 60 minutos.

El $72 \%$ de los procedimientos quirúrgicos que se realizan en el HUSJ son llevados a cabo por solo cinco especialidades, que en su orden son cirugía general, cirugía plástica, ginecología, traumatología y urología. En contraste con un gran estudio realizado en Brasil por Giordani et al, la especialidad que más operó fue cirugía ortopédica (25\%), seguida de cirugía general con $24 \%{ }^{(5)}$. En otro reporte realizado en India por Bhasin et al ${ }^{(1)}$, ginecobstetrícia fue el servicio que operó con mayor frecuencia con $32,4 \%$ seguido de cirugía general con $29,3 \%$. La distribución del tipo de especialidad predominante responde a necesidades locales y regionales de atención así como a la disponibilidad de capacidades hospitalarias.

La cirugía realizada con mayor frecuencia en el estudio de Bhasin et al ${ }^{(1)}$, fue la cesárea con 3,32\%, a esta le siguieron: operación de cataratas $1,41 \%$; derivación cardiaca $1,05 \%$; histerectomía $1,05 \%$ y apendicectomía con $0,95 \%$. La frecuencia de cesáreas realizadas en el HUSJ convierten este procedimiento en el más realizado. Para tener un contexto más cercano, en el estudio de Londoño et al ${ }^{(2)}$ realizado en Armenia (Colombia), el procedimiento más frecuentemente realizado fue la herniorrafía con una prevalencia de 43,8\% seguido de la safeno-varicectomía con $36,5 \%$. Nuevamente las diferencias se explican por los niveles de complejidad y atención. Sin embargo, la distribución de los procedimientos que se realizan con más frecuencia podría ser de gran interés clínico y académico. Su información permite replantear las patologías con énfasis en la enseñanza en tercer nivel de atención y el orden de entrenamiento quirúrgico para residentes y estudiantes de ciencias de la salud.

Las cirugías provenientes de hospitalización, urgencias y cirugía ambulatoria se distribuyen de forma equitativa en el HUSJ. El hospital cuenta con siete quirófanos de los cuales solo uno corresponde a urgencias de 24 horas. De acuerdo a los porcentajes obtenidos anteriormente, se puede inferir que el área de urgencias debe tener a su disposición un tercio del tiempo quirúrgico o al menos dedicado a esta actividad. Estas proporciones guardan cierto acuerdo con otros estudios. En Giordani et al ${ }^{(5)}$ la cirugía de urgencia se presentó en un $31 \%$ y la electiva en un $69 \%$. En el estudio de Bhasin et al ${ }^{(1)}$ realizado en Nueva Delhi, el 38.3\% corresponde a urgencias y el $61,7 \%$ fue electiva. (En los estudios revisados dentro de la categoría electiva, se encuentran integradas las categorías de hospitalización y ambulatoria, que están por separado en nuestro estudio).

La mayor cantidad de pacientes operados en HUSJ son adultos pertenecientes al régimen subsidiado los cuales componen más de la mitad de usuarios. No se encontraron estudios similares con información relacionada en Colombia. Este hallazgo tiene relación con el perfil de pacientes atendidos en el HUSJ en donde la mayor proporción de pacientes atendidos son del régimen subsidiado.
Como último punto, al momento de describir variables quirúrgicas, uno de los datos que resulta relevante mencionar es la duración de los procedimientos quirúrgicos por impactos económicos, administrativos, organizacionales y clínicos. En los resultados del presente estudio se encontró que la media de la duración quirúrgica es de 58 minutos, semejante a lo descrito en el estudio brasileño realizado por Giordani et al que evidenció una media de 51,6 minutos para la duración de procedimientos quirúrgicos ${ }^{(5)}$. La mediana de duración global (que presenta un resumen robusto del tiempo sin influencias de los procedimientos muy largos o muy cortos) para el hospital es de 60 minutos.

Por último se determinó que el mes de julio es cuando más cirugías se llevan a cabo pues se realizaron, aproximadamente, la décima parte de todas las cirugías del año. En el mes de febrero se realizó una menor cantidad de cirugías. En nuestra interpretación de los datos, estas variaciones responden a circunstancias principalmente administrativas y de contratación en el tiempo. De la misma forma, los días viernes y sábados la utilización de los quirófanos es menor que otros días de la semana posiblemente por saturación de la capacidad hospitalaria. La organización administrativa juiciosa del proceso quirúrgico contemplando la relación demanda y oferta garantiza el funcionamiento efectivo y evita cancelaciones quirúrgicas ${ }^{(6)}$.

Este estudio sorteó diversas limitaciones, y por su naturaleza observacional, sus resultados fueron analizados de forma crítica. Cada paciente contaba con múltiples diagnósticos y múltiples intervenciones, sin embargo para el análisis de los datos únicamente se consideró el primer diagnóstico (anotado como principal) y el primer procedimiento realizado sin analizar si era el más relevante en cada contexto individual. En estudios que utilizan información alojada en datos previamente diligenciados generalmente presentan sesgos de información que en este caso intentaron minimizarse. Futuros esfuerzos de investigación en esta área deberían garantizar la calidad y validez de los datos recolectados (tiempos quirúrgicos, horarios, diagnósticos, intervenciones quirúrgicas, razones de cancelación quirúrgica), apropiadas clasificaciones diagnósticas y de las intervenciones quirúrgicas y valoración de los desenlaces a mediano y largo plazo de los procesos quirúrgicos hospitalarios.

\section{Conclusión}

El perfil epidemiológico quirúrgico del HUSJ en el año 2014 tiene concordancia con reportes de otras latitudes para niveles de complejidad similar. Producto de este análisis, existen oportunidades de mejoramiento en la prestación y ejecución del servicio quirúrgico. Entre ellas, de acuerdo a la demanda, la asignación del quirófano de urgencias y la distribución de salas de acuerdo a especialidad y tipo de cirugía.

\section{Referencias}

1. BHASIN SK, ROY R, AGRAWAL S, SHARMA R. An Epidemiological Study of Major Surgical Procedures in 
an Urban Population of East Delhi. Indian J Surg. $2011 ; 73(2): 131-5$.

2. LONDOÑO A, MORALES J, MURILLAS M. Características epidemiológicas y factores de riesgo relacionados con la infección en el sitio operatorio en procedimientos de cirugía general. Rev Chil Cirugía. 2011;63(6): 559-65.

3. BELTRÁN BELTRÁN JE, BUITRAGO PÉREZ J DEL P, REYES HERRERA AC. Descripción y analisis del perfil epidemiológico, la demanda y la oferta del servicio de gineco obstetricia del Hospital Universitario San Ignacio. (Tesis de posgrado). Bogotá: Universidad EAN; 2010.
4. R DEVELOPMENT CORE TEAM, R: A Language and Environment for Statistical Computing. (sitio en internet) (2011). Vienna, Austria: The R Foundation for Statistical Computing. ISBN: 3-900051-07-0. Disponible en http:/ /www.R-project.org/.

5. GIORDANI AT, SONOBE HM, EZAIAS GM, VALÉRIO MA, BARRA MR, STADLER DV. Perfil De Pacientes Qirúrgicos Atendidos En Un Hospital Público. Rev enferm UFPE line. 2015;9(1):54-61.

6. MUÑOZ A, SARMIENTO CP, TORRES MS. Cancelación de cirugía programada en una institución de III nivel de atención. Rev Fac Ciencias de la Salud, Universidad del Cauca. $2011 ; 13(3): 10-15$. 